\title{
Cosmetic Acts and Frontal Cortex Function in Upper Middle-aged Japanese Women
}

\author{
Taketoshi Hatta ${ }^{1, *}$, Takeshi Hatta ${ }^{2}$ \\ ${ }^{1}$ Gifu University of Medical Sciences, 795-1, Hiraga-Nagamine, Seki City, Gifu, 501-3892, Japan \\ ${ }^{2}$ Kansai University of Welfare Sciences, 3-11, Asahigaoka, Kashiwara City, Osaka 582-0026, Japan
}

\begin{abstract}
This study examined the relationship between cosmetic acts and frontal cortical cognitive function of Japanese upper middle-aged women. Cosmetic habit of the participants was addressed as an important part of social function in women. Two hundred and six community dwellers were given both the questionnaire for cosmetic acts and the cognitive assessment battery as a part of Yakumo Study. The results showed that prefrontal cortex related cognitive performances for women who were sustaining the habitual cosmetic acts (both skin care and cosmetic regimes) were better than those of women who have already abandoned the habit. This suggests an important relation between maintaining cosmetic acts even in elderly and sustaining frontal cortex function.
\end{abstract}

Keywords Cosmetic Acts, Aging, Social Vulnerability, Frontal Cortex Function, Japanese Women

\section{Introduction}

From the middle stage of our lives, a substantial decay is observed in physical functions, such as a slower walking pace and difficulty in running up steps. This phenomenon is also the case in cognitive function, and can be seen in several cognitive abilities such as memory loss and difficulty in learning new topics, etc. Many evidences on so-called 'cognitive aging' have been accumulated, describing developmental phenomena and suggesting ways of preserving cognitive abilities even in the later stages of life (e.g., Andrew, Fisk, \& Rockwood, 2011; Cavanough \& Blanchard-fields, 2006; Cabeza, Nyberg, \& Park 2005; Hampshire, Gruszka, Fallon, \& Owen, 2008; Karasawa \&Hatta, 2009; Motes, Biswl, \& Rypma, 2011; Park, \& Schwarz, 2000; Prof, Paillard-Borg, \& Winblad, 2004; Praksh, Erickson, Colcombe, Kim, Voss, \& Kramer, 2009; Paxton, Barch, Racine, \& Braver, 2008; Singh-Manoux, Marmot, Glymour, Sabia, Kivimaki, \& Durravot, 2011; Stern, 2007; Tucker-Drob, Johnson, \& Jones, 2009).

In short, most of these studies indicate that the effects of aging on working memory, perceptual speed, attention, and other various cognitive ability decline appear from around age of 60 years old, and a person's life style in middle age, including physical activities and daily cognitive habit (e.g., reading, writing, attend club activities, etc.) relates strongly to a sustainability of cognitive abilities in the later stages of life. Recent review work by Andrew, Fisk, and Rockwood

* Corresponding author:

hatta@u-gifu-ms.ac.jp (Taketoshi Hatta)

Published online at http://journal.sapub.org/ijpbs

Copyright (C) 2011 Scientific \& Academic Publishing. All Rights Reserved
(2011) supported these proposals in their own words; social vulnerability relates to prefrontal cortex function.

The present study focused on the relation between everyday life habit of cosmetic acts and cognitive abilities in Japanese upper middle-aged women. This is because those cosmetic acts are regarded one of the most important social activities for adult women, especially in Japanese, to attend social situation such as meet friends, leisure activities, etc. As far as we know, few studies have reported substantial relation between cosmetic acts and cognitive aging until now.

It is assumed that cosmetic acts (skin care, lip stick, drawn eye brow and other regimes) relates to frontal cortex function, because not only it is an important social activity but also cosmetic acts require temporal order planning, sustaining attention to the act that involves vision-finger/hand motor coordination such as apply lipstick wisely on proper lip location, finely draw eye brew, etc. (Hatta, Iwahara, Karasawa, \& Hatta, 2007; Spreen \& Strauss, 1998). Further, women suffering senile dementia do not continue the cosmetic habit, while those who are suffering depression usually refrain from cosmetic acts due to a lack of desire relating to frontal cortex function (Abe, 1992; Miller, \& Cox, 1982; Yogo, et al., 1990). Therefore, it is hypothesized that to sustain cosmetic acts relates to preserve frontal cortex function of elderly women.

Japanese women of $94 \%$ start cosmetic acts from their mid-teens and continue the practice for a long period (Abe, 2004). Needless to say, cosmetic acts depend on cultural style and it may not be an exaggeration to say that most Japanese women in their 20s pun on make up every day, while most European women may put on make-up on special occasion. According to the survey by Shiseido Company, 
more than $97 \%$ of 4903 Japanese women who live in Tokyo area (age range from 15 to 64 years) had an experience to engage in cosmetic acts (Shimoda, 1993). This also indicated that women begin skincare as the first stage of the acts and subsequently move on to make-up such as rouge, lipstick and mascara, which are applied in order to enhance their appearance. Based on these Shiseido survey, the cosmetic acts of Japanese elderly women can be classified into three groups, that is, no cosmetic act (Nothing group), skincare only (Skincare group), skincare plus deeper make-up (Make-up group).Recent questionnaire survey (Hatta, Iwahara, Karasawa, \& Hatta, 2007) reported that $89 \%$ of 314 women aged from 40 to 91 years old make skin care every day even they have no idea to visit outside $(86 \%$ of them conduct cosmetic acts full regimes such as skin care, lip sticks, eye line, blush, and plus alpha on the occasion to meet friend).

In light of these findings, we examined three issues for the relation between cosmetic acts and cognitive aging. First was to overview cosmetic activity in Japanese elderly women who live in a rural region of Japan. Second issue was to examine the relation between frontal cortex function and cosmetic acts as an index of social vulnerability, i.e., working hypothesis was that Skincare and Makeup groups(women who are continuing cosmetic acts) show better performance than Nothing group (women who have abandoned their cosmetic habit) in test relating to frontal cortex function. Third was to examine cosmetic activity in elderly more precisely, i.e., working hypothesis was that Make-up group who is continuing high level of cosmetic acts (skincare plus deeper make-up) shows better performance than Skincare only group who is keeping low level of cosmetic acts (skincare and just lipstick use but abandon anymore make-up acts) in cognitive tasks. This is because Makeup group requires cosmetic actions consisting of higher levels of cognitive components than Skincare group. The makeup involves precise vision-finger/hand motor coordination to apply lipstick wisely on proper lip location, finely draw eye brew, etc. These require cognitive components such as planning, temporal order, attention, spatial coordination, etc., whereas skincare action requires only rough vision-finger/hand motor coordination to wash her face.

Some may claim that not only cognitive decline but also various factors, such as deficit of vision and motor function, economic status, IQ, depression, relates to quit from acquired cosmetic habit. These confounding factors are controlled as far as we can in this study sample. Economic status variation is small (agriculture, forestry and fishery are major industries of this cohort study, Yakumo Study) and dementia or depression suspects are excluded from the analysis.

\section{Method}

\subsection{Participants}

The participants were 206 women living in Yakumo Town in Japan. Therefore, this study have benefits from a relatively homogeneous sample of women from the same town in Japan and who presumably have a similar socioeconomic status with regards to their employment and living style. Table 1 shows participants' demographic data.

They completed the Nagoya University Cognitive Assessment Battery (NU-CAB), as well as the questionnaire on cosmetic acts. The NU-CAB consists of a part of Yakumo Study, and the questionnaire for everyday life cosmetic acts was given in advance. The age range was 55 to 91 years, and the average age was $66.44(\mathrm{SD}=8.14)$ years old. The subjects showed no signs of any neuropsychological disorders such as paralysis, motor function or senile dementia. Originally, 231 women participated in this study, however the data of 25 participants were excluded by the reason of dementia-suspected and personal discipline of anti-cosmetic act and quitted already in early age due to skin trouble. All participants started their cosmetic acts when they left from school, though the exact date of the beginning was unknown, but all participants answered that they have begun cosmetic act during the age of 16 to 20 years old. The Shiseido survey reported that it is a general Japanese custom that female started cosmetic behaviour after school age and it was confirmed by the questionnaire.

\subsection{Measures}

Cosmetic acts habit: The questionnaire was sent to each participant 3 weeks before the NU-CAB testing and they were asked to bring the questionnaires if they were able to participate in our research project. The questionnaire was collected at the reception desk of the health screening venue of the Yakumo Study on that day. The data analysed were based on daily cosmetic acts on the day without any special plans to go outside. The participants were asked to fill in the appropriate "yes" or "no" box of the questionnaire sheet relating to their cosmetic acts.

\subsection{Cognitive Assessment}

The NU-CAB addressed mainly to the examination of frontal cortex related abilities, such as attention, language, memory, working memory and executive functions (Hatta, 2003). Both reliability and validity have been reported elsewhere (Hatta, 2004; Hatta, Nagahara, Iwahara, \& Ito, 2005; Ito \& Hatta, 2006; Hatta, et. al., 2008). The NU-CAB consisted of test items such as MMSE, Logical Memory Test (Japanese version of Wechsler Memory Scale), Money Road Test, Stroop Test, D-CAT (digit cancellation test) and Verbal Fluency Test (letter fluency test; LFT and semantic fluency test: SFT). In particular, the validity examination by means of the NIRS (Near-infrared Spectroscopy) identified robust activation of prefrontal cortex in the D-CAT and LFT, and both frontal and temporal cortices activation in the SFT and the Logical Memory Test. Among NU-CAB items, we used 9 measures (MMSE, Logical memory, Money road test, Stroop (color name reading, and letter naming), D-CAT (1 and 3 letters cancellation conditions), LFT, and SFT to ad- 
dress our research objectives.

\section{Results}

Table 1 shows the results of the questionnaire for participants' cosmetic acts and performances in cognitive test items. As for the first issue, 175 women responded "yes". This means they habitually engaged in cosmetic act, while 31 women responded "no". It showed that $84.9 \%$ of participants are engaging in some kinds of cosmetic acts in everyday life.

To examine the second issue, three groups, Nothing group, Skincare group, and Makeup group were classified. There was no participant who made makeup without skincare.

As the performance scores have different ranges, all performance score were normalized and then an analysis of variance was conducted. An analysis of variance (group $\times$ cognitive test item) showed a significant interaction between two main factors $(\mathrm{F} 2,8=3.96, \mathrm{p}<0.001)$. As the interaction was significant, further analyses were conducted for each item. The results revealed that there was significant group difference in three items (Logical memory (F2, $203=$ 4.074, $\mathrm{p}<0.018$ ), Stroop dot naming (F2, $203=6.608, \mathrm{p}<$ 0.002). Stroop letter naming $(F 2,203=10.185, p<0.001))$ and four items (MMSE $(F 2,203=2.600, p<0.07)$, D-CAT 1 digit $(\mathrm{F} 2,203=2.344, \mathrm{p}<0.09)$, D-CAT 3 digits $(\mathrm{F} 2,203=$ $2.403, \mathrm{p}<0.09)$, LFT $(\mathrm{F} 2,203)=1.84, \mathrm{p}<0.16))$ showed a tendency of group difference. Three items (Money road test $(p<0.549)$ and SFT $(p<0.569)$ did not show group difference. Further examinations showed that all significant difference reflected difference between Nothing group and other two groups, i.e., any significant difference was not shown between Skin care group and Makeup group for task items.

Table 1. Demographic Data and Mean Performances in Cognitive Tasks. (SDs are shown in parentheses).

\begin{tabular}{|c|c|c|c|}
\hline Group & Nothing & $\begin{array}{c}\text { Just Skin- } \\
\text { care }\end{array}$ & $\begin{array}{c}\text { Skincare } \\
\text { Plus } \\
\text { Make-up }\end{array}$ \\
\hline Participants (N) & 31 & 83 & 92 \\
\hline Mean Age & $68.16(9.01)$ & $66.08(8.71)$ & $65.64(7.24)$ \\
\hline $\begin{array}{c}\text { MMSE } \\
\text { (Mini-mental State) }\end{array}$ & $26.32(2.34)$ & $26.83(2.45)$ & $27.37(2.30)$ \\
\hline Logical Memory & $12.13(4.43)$ & $13.55(6.35)$ & $15.24(5.44)$ \\
\hline Money Road Test & $8.74(2.52)$ & $9.06(2.73)$ & $9.30(2.40)$ \\
\hline $\begin{array}{c}\text { Stroop (Dot Color } \\
\text { Naming) }\end{array}$ & $35.00(8.12)$ & $30.54(7.24)$ & $29.60(6.83)$ \\
\hline $\begin{array}{c}\text { Stroop (Letter } \\
\text { Naming) }\end{array}$ & $59.96(31.35)$ & $45.33(15.07)$ & $43.38(14.01)$ \\
\hline D-CAT (1 digit) & $22.10(5.42)$ & $24.76(7.08)$ & $25.11(7.01)$ \\
\hline D-CAT (3 digits) & $36.65(11.85)$ & $41.76(12.51)$ & $41.59(11.04)$ \\
\hline Letter Fluency Test & $6.94(3.33)$ & $8.25(4.27)$ & $8.32(3.04)$ \\
\hline $\begin{array}{c}\text { Semantic Fluency } \\
\text { Test }\end{array}$ & $12.52(6.17)$ & $13.43(4.85)$ & $12.82(4.12)$ \\
\hline
\end{tabular}

These results support the hypothesis based on the second issue that women who quitted from habitual cosmetic acts showed inferior performances in certain number of items that are regarded substantial relation to the prefrontal cortex, and frontal and temporal cortex function. It may conclude that elderly women who are continuing cosmetic acts in everyday life are sustaining frontal cortex related cognitive function.

Third issue was not supported. No item showed a significant difference between Skincare and Makeup groups.

\section{Discussion}

The relation between social activity (we especially focused on the habitual cosmetic acts as one of the important social activities) and frontal cortex function in elderly was investigated, as this kind of systematic examination has not been reported (Andrew, Fisk, \& Rockwood, 2011; Tucker-Drob, Johnson, \& Jones, 2009).

Our results revealed firstly that the population of Japanese women who are continuing cosmetic acts (skin care only and skincare plus makeup) was $84 \%$ and it was much larger than Inami and Hama (2000), though it was slightly smaller than Shiseido survey (Shimoda, 1993). Inami and Hama (2000) reported that $39 \%$ of women in 60 's and $54 \%$ in 70's quit from habitual cosmetic acts. According to their reports for elderly, some abandoned the habitual use of cosmetics after marriage or childbirth, while others continued the habit. Although our participants are living in a typical Japanese rural area (Yakumo town is an agricultural and fishing village in Hokkaido Island), cosmetic acts participation was increased, compared with urban population in their report. This may be a cohort effect and the reflection of increasing income and media advertisement for cosmetic acts for elderly women.

The second issue relates to the question whether cosmetic acts keeping relate to subserve frontal cortex function. Based upon the cognitive aging data from Yakumo Study (Aoki \& Ito, 1999), Hatta $(2003,2009)$ proposed a cognitive aging model that cognitive abilities acquired at a late stage of human development are more vulnerable than those acquired at an earlier stage. This proposal is basically in the same vein with disuse theory of cognitive aging by Salthouse (1991). Prof, Paillard-Borg, and Winblad (2004) supported the proposal by Salthouse. In accord with these previous aging studies, Andrew, Fisk, and Rockwood (2011) summarized that social vulnerability relate to prefrontal cortex function in elderly. They stressed importance to engage social activities (e.g., leisure) to sustain prefrontal cortex function.

The results showed that the second issue was supported as the women who are continuing cosmetic acts showed better performance in frontal cortex related function than women who have abandoned their cosmetic acts. The Stroop test and Logical memory test showed highly significant group difference and MMSE, D-CAT and LFT showed a supportive tendency, while Money road test and SFT failed to show group difference.

Concerning to cognitive mechanisms for Stroop test, and D-CAT, a strong engagement of the frontal cortex has been suggested (Awh \& Jonides, 2001; MacLeod \& MacDonald, 2000; Mead, et al, 2002; Paresh, et al., 2005), as these tests 
require attention focusing, attention retention and response inhibition. Further, Logical memory test and MMSE seem to reflect frontal cortex and temporal cortex engagement. For LFT, NIRS (Near-Infrared Spectroscopy) showed evidence of a stronger activation in the prefrontal cortex in the LFT than SFT, while SFT has more engagement than LFT in temporal cortex (Ito, et al, 2004; Ito \& Hatta, 2006; Hatta, et al., 2009).

These seem to induce the results that Nothing group showed poorer performances in frontal cortex related cognitive function. On the other hand, both Money Road Test and SFT failed to show a significant group difference. These suggest that group difference was appeared only in the task depending primarily on the prefrontal area, but it was not the case in the task depending primarily on the right temporal-parietal cortex (Money Road Test), and temporal areas (SFT).

The third issue that predicted a difference such that Makeup group shows superior performance than Skincare group as well as Nothing group was not supported. Two things might reflect this. First is that acquiring stage of skincare and makeup in cosmetic action might not be different (Shimoda, 1993). Hatta (2006) stressed an importance of acquiring order of cognitive acts in human development in cognitive aging model but both acts acquired at same time. This might be a reason why no substantial difference between Makeup and Skincare groups was shown. Second is that while actions of Skincare and Makeup groups differ, the both commonly involve awareness of "should be self" or "conscious of others" in social situation. To aware of objective self and others is crucially important to preserve frontal cortex function properly (not a level of cosmetic acts) and this awareness might be a key for the decline of social vulnerability in elderly and might be a reason why Skincare and Makeup groups did not differ.

\section{Conclusions}

The present study revealed a relation between cosmetic acts and frontal cortex function in elderly, and it was suggested that to keep on social activity including cosmetic acts in later stage of life is an important factor to sustain cognitive functions, at least for Japanese women. To strengthen this tentative conclusion, further examinations that would include more precise individual information for more concrete cosmetic acts is necessary, probably by means of concrete and intensive interviews of individuals.

\section{ACKNOWLEDGEMENTS}

This study was supported by the grant for science research to the first author from the Ministry of Education, Science, Sports and Culture in Japan (No.19330158, No. 20390397) and the grant to both authors from the Kose Cosmetic Science Research Foundation (2007). The authors thank lot to
Mrs. Elizabeth Segal for her kind assistance of English description.

\section{REFERENCES}

[1] Abe, T., 1992, Psychology of cosmetics. Fragment. Journal. 20, 55-61

[2] Abe, T., 2004, Psychological Studies of Skincare in Japan: A Review. Tohoku Psychological. Folia. 63, 53-60.S

[3] Andrew, M. K., Fisk, J. D., and Rockwood, K., 2011, Social vulnerability and prefrontal cortical function in elderly people: A report from the Canadian Study of health and aging. International Psychogeriatric, 23, 450-458

[4] Aoki, K., and Ito, Y., 1999, Reports on Y-Town cohort project with Nagoya University Medical School. Yakumo Town

[5] Awh, E., and Jonides, J., 2001, Overlapping mechanisms of attention bans spatial working memory. Trends in Cognitive Science, 5, 119-126

[6] Cabeza, R., Nyberg, L., and Park, D., 2005, Cognitive Neuroscience of Aging. Oxford: Oxford University Press

[7] Cavanough, J. C., \& Blanchard-fields, F., 2006, Adult development and aging (5th edition). Belmont: Wadsworth

[8] Hampshire, A., Gruszka, A., Fallon, S. J., and Owen, A. M., 2008. Inefficiency in self-organized attentional switching in the normal aging population is associated with decreased activity in the ventrolateral prefrontal cortex, Journal of Cognitive Neuroscience, 20, 1670-1686

[9] Hatta, T., 2003, Brain and Behavior. Tokyo: Ishiyaku-syuppan

[10] Hatta, T., 2004, Development of cognitive assessment battery (NU-CAB) for community health examination. Journal of Human Environmental Studies, 2, 15-20

[11] Hatta, T., 2009, How to sustain cognitive abilities in aged people. In K. Karasawa and T. Hatta (Eds.) Happy aged life. Kyoto: Nakanishiya

[12] Hatta, T., Iwahara, A., Karasawa, K., and Hatta, T., 2007, The cosmetic behavior and higher brain function of middle and old women. Journal of Human Environmental Studies 5, $35-40$

[13] Hatta, T., Kanari, A., Mase, M., Kabasawa, H., Ogawa, Shirataki, T., Hibino, S., Iida, A., Nagano, Y., Abe, J., and Yamada, K., 2008, Brain mechanisms in Japanese verbal fluency test: Evidence from examination by NIRS (Near-Infrared Spectroscopy), Asia-Pacific Journal of Speech, Language and Hearing, 11, 103-110

[14] Hatta, T., Nagahara, N., Iwahara, A., and Ito, E., 2005, Three word recall and logical memory in normal aging. Journal of Human Environmental Studies, 3, 7-12

[15] Hatta, T., Kanari, A., Mase, M., Nagano, Y., Shirataki, T., and Hibino, S., 2009, Strategy effects on word searching in Japanese letter fluency tests: Evidence from the NIRS findings. Read \& Writing, 22, 1041-1052 
[16] Karasawa, K., and Hatta, T., 2009, Happy aged life. Kyoto: Nakanishiya Press

[17] Kimura, D., 1996, Sex and cognition. Cambridge; MIT Press

[18] Inami, K., and Hama, H., 2000, Aged female and cosmetic behavior. Journal of Textual Machinery Society of Japan, 53, 222-228

[19] Ito, E., and Hatta, T., 2006, Reliability and validity of verbal fluency tasks. Japanese Journal of Neuropsychology, 22, 146-152

[20] Ito, E., Hatta, T., Ito, Y., Kogure, T., and Watanabe, H., 2004, Performance of verbal fluency tasks in Japanese healthy adults. Japanese Journal of Neuropsychology, 20, 254-263

[21] MacLeod, C. M., and Mac Donald, P. A., 2000, Interdimensional interference in the Stroop effect: Uncovering the cognitive and neural anatomy of attention. Trends in Cognitive Science. 4, 383-391

[22] Mead, L. A., Mayer, A. R., Bobholz, J. A., Woodley, S. J., Cunning ham, J. M., and Hammeke, T. A., 2002, Neural basis of the Stroop interference task: Response competition or selective attention. Journal of International Neuropsychological. Society, 8, 735-742

[23] Miller, L., and Cox, C. L., 1982, For appearance sake; Public self-consciousness and makeup use. Personality Social Psychology Bulletin. 8, 748-751

[24] Motes, M. A., Biswal, B. B., and Rypma, B., 2011, Age-related relationships between prefrontal cortex activation and processing efficiency. Cognitive Neuroscience, 2, $1-10$

[25] Paresh, M., Rolf, H., Andrew, O., Richard, G., Diane, M., martin, M., Jon, D., and Masud, H., 2005, Spatial working memory capacity in unilateral neglect. Brain, 128, 424-435
[26] Park, D. C., and Schwarz, N., 2000, Cognitive aging: A primer. Hove: Psychology Press

[27] Paxton, J. L., Barch, D. M., Racine, C. A., \& Braver, T. S. (2008). Cognitive control, goal maintenance, and prefrontal function in healthy aging. Cerebral Cortex, 18, 1010-1028

[28] Prakash, R. S., Erickson, K. I., Colcombe, S. J., Kim, J. S., Voss, M. W., and Kramer, A. F., 2009, Age-related differences in the involvement of the prefrontal cortex in attentional control. Brain \&Cognition, 71 328-335

[29] Prof, L. F., Paillard-Borg, S., and Winblad, B., 2004, An active and socially integrated lifestyle in later life might protect against dementia, Lancet Neurology, 3, 343-353

[30] Salthouse, T. A. 1991, Theoretical perspectives on cognitive aging. Hillsdale: Erlbaum Associates

[31] Shimoda, M. 1993, Usability of makeup water. In Shiseido (Ed.) Psychology of Cosmetics, Tokyo: Fragrance Journal Press. Pp.59-65

[32] Singh-Manoux, A., Marmot, M. G., Glymour, M., Sabia, S., Kivimaki, M., \& Dugravot, A., 2011, Does cognitive reserve shape cognitive decline? Annals of Neurology, 70 296-304

[33] Spreen, O., and Strauss, E., 1998, A compendium of neuropsychological tests. Oxford: Oxford University Press

[34] Stern, Y., 2007, Cognitive reserve: Theory and application. New York: Taylor \& Francis

[35] Tucker-Drab, E., Johnson, K. E., and Jones, R. N., 2009, The cognitive reserve hypothesis: A longitudinal examination of age-associated declines in reasoning and processing speed. Developmental Psychology, 45 431-446

[36] Yogo, M., Hama, H., Tsuda, K., Suzuki, Y., and Tagai, K., 1990, Effects of cosmetic behavior on psychological health of women. Health Psychology Research. 3, 28-32 